\title{
Brain systems underlying encounter expectancy bias in spider phobia
}

\author{
Tatjana Aue • Marie-Eve Hoeppli • Camille Piguet • \\ Christoph Hofstetter • Sebastian W. Rieger • \\ Patrik Vuilleumier
}

Published online: 19 February 2015

(C) Psychonomic Society, Inc. 2015

\begin{abstract}
Spider-phobic individuals are characterized by exaggerated expectancies to be faced with spiders (so-called encounter expectancy bias). Whereas phobic responses have been linked to brain systems mediating fear, little is known about how the recruitment of these systems relates to exaggerated expectancies of threat. We used fMRI to examine spiderphobic and control participants while they imagined visiting different locations in a forest after having received background information about the likelihood of encountering different animals (spiders, snakes, and birds) at these locations. Critically, imagined encounter expectancies modulated brain responses differently in phobics as compared with controls. Phobics displayed stronger negative modulation of activity in the lateral prefrontal cortex, precuneus, and visual cortex by encounter expectancies for spiders, relative to snakes or birds (within-participants analysis); these effects were not seen in
\end{abstract}

Electronic supplementary material The online version of this article (doi:10.3758/s13415-015-0339-6) contains supplementary material, which is available to authorized users.

T. Aue $(\bowtie) \cdot$ C. Hofstetter $\cdot$ S. W. Rieger $\cdot$ P. Vuilleumier Swiss Center for Affective Sciences, University of Geneva, Chemin des Mines 9, 1202 Geneva, Switzerland

e-mail: tatjana.aue@unige.ch

T. Aue $\cdot$ C. Piguet $\cdot$ C. Hofstetter $\cdot$ P. Vuilleumier

Department of Neuroscience, University Medical Center, University of Geneva, Rue Michel-Servet 1, 1211 Geneva, Switzerland

T. Aue $\cdot$ C. Piguet $\cdot$ C. Hofstetter $\cdot$ S. W. Rieger $\cdot$ P. Vuilleumier Geneva Neuroscience Center, University of Geneva, Rue Michel-Servet 1, 1211 Geneva, Switzerland

M.-E. Hoeppli

Alan Edwards Centre for Research on Pain, McGill University, \#M/

19, 3640 University Street, Montreal, Quebec H3A 0C7, Canada

C. Piguet

Department of Mental Health and Psychiatry, University of Geneva

Hospitals, Rue de Lausanne 20, 1201 Geneva, Switzerland controls. Between-participants correlation analyses within the phobic group further corroborated the hypothesis that these phobia-specific modulations may underlie irrationality in encounter expectancies (deviations of encounter expectancies from objective background information) in spider phobia; the greater the negative modulation a phobic participant displayed in the lateral prefrontal cortex, precuneus, and visual cortex, the stronger was her bias in encounter expectancies for spiders. Interestingly, irrationality in expectancies reflected in frontal areas relied on right rather than left hemispheric deactivations. Our data accord with the idea that expectancy biases in spider phobia may reflect deficiencies in cognitive control and contextual integration that are mediated by right frontal and parietal areas.

Keywords Spider phobia $\cdot$ Fear $\cdot$ Risk estimation $\cdot$ Encounter expectancy bias · fMRI

Spider phobia is frequent in the general population (lifetime prevalence: $11 \%$; point prevalence: $3.5 \%$; Fredrikson, Annas, Fischer, \& Wik, 1996) and may have an evolutionary origin (Öhman \& Mineka, 2001). However, while biological preparedness may be at the origin of animal phobia, not all of us are similarly frightened by actual or imagined encounters with fear-relevant animals, such as spiders and snakes. This may be related to different expectancies regarding these potential sources of threat (e.g., Davey \& Dixon, 1996).

We (Aue \& Hoeppli, 2012) have argued that elevated subjective expectancies of threat (regarding either the likelihood of an encounter with the feared animal, or the likelihood of incurring negative consequences from such encounters, or both) may increase an event's salience and render threatrelated information more readily available. Hence, biased (i.e., irrational) expectancies in phobia may be causally related 
to some of the observed biases in attention and memory (e.g., Mogg \& Bradley, 2006; Olatunji, Sawchuk, Lee, Lohr, \& Tolin, 2008; Watts, McKenna, Sharrock, \& Trezise, 1986; see Bar-Haim, Lamy, Pergamin, Bakermans-Kranenburg, \& van IJzendorn, 2007, and Mitte, 2008, for meta-analytic data). Important insight into the mechanisms underlying expectancy bias in spider phobia can be gained by studying this phenomenon with brain imaging techniques. ${ }^{1}$ Yet, the majority of neurocognitive studies on spider-phobic or spider-fearful individuals have focused on particularities in perceptual/ attentional and memory processes, while overlooking particularities in subjective expectations with respect to spiders.

Standard presentation paradigms, in which phobic or fearful individuals watched pictures or films - without simultaneous assessment of expectancies - showed that spider phobia correlates with enhanced activity of the fear circuit (e.g., Davis \& Lang, 2003; LeDoux, 1996) and with reduced activity in emotion regulation networks (e.g., Carlsson et al., 2004; Hermann et al., 2009), including regions such as the insula, medial prefrontal cortex (mPFC), and lateral prefrontal cortex (IPFC).

Carlsson et al. (2004), for instance, found that conscious perception of animal phobia-related pictures led to selective activation in the amygdala, anterior insula, medial orbitofrontal cortex (mOFC), anterior cingulate cortex (ACC), and periaqueductal gray (see also Dilger et al., 2003; Rauch et al., 1995), activations that were not seen for evolutionary fear-relevant but non-phobogenic animals. At the same time, activity was reduced in both the dorsolateral prefrontal cortex (dlPFC) and the right lateral OFC, when phobogenic trials were contrasted with fear-relevant trials. Together, these changes have been suggested to signal increased activation of the fear circuit and defective top-down control for phobia-related stimulus material.

Somewhat contradictory to these findings, though, are those of an fMRI study by Paquette et al. (2003), which revealed activation in the right dIPFC when spider phobics viewed film excerpts depicting spiders (cf. Schienle, Schäfer, Walter, Stark, \& Vaitl, 2005), which the authors attributed to the use of metacognitive strategies for the regulation of fear, possibly mediating avoidance behavior. After completion of cognitive behavior therapy, no such activation was found (for further changes associated with cognitive behavior therapy of spider phobia, see Schienle, Schäfer, Hermann, Rohrmann, \& Vaitl, 2007). To solve the discrepancy regarding the dIPFC activations in animal phobia, it has been proposed that both non-phobics and phobics use top-down control, but perhaps of different kinds or with different effectiveness.

In sum, some caution is warranted because prefrontal activations are not reliably observed across different studies (see

\footnotetext{
${ }^{1}$ See Aue, Lavelle, and Cacioppo (2009) for the utility of fMRI in the investigation of psychological phenomena.
}

Etkin \& Wager, 2007). It is noteworthy as well that several earlier studies did not reveal any difference in amygdala activity even between phobia-related and neutral stimulus material (e.g., Fredrikson et al., 1993; Paquette et al., 2003; Wik et al., 1993). Dilger et al. (2003) argued that this may be due to either limited temporal resolution of PET or the use of block designs. Nevertheless, a consistent emerging picture is that individuals with animal phobia generally exhibit hyperactivation of the subcortical fear circuits, associated with various anomalies (most often a decrease) in the recruitment of control systems when confronted with phobia-relevant cues. However, more research is clearly required to clarify which components in these neural networks are more specifically related to particularities in subjective expectancies. Parts of the phobiaspecific brain responses that reappear across diverse studies that use different experimental paradigms may indicate deviated expectancy processes. Identification of commonalities across studies and the link between these (de)activations and phobogenic expectancies may be helpful in the identification of situations that characterize irrationality in expectancies; a discovery that could have far-reaching implications for therapeutic applications.

The current study's focus, thus, is on the encounter expectancy bias in spider phobia, that is, the tendency to overestimate the likelihood of encountering spiders across a variety of situations (Aue \& Hoeppli, 2012; de Jong \& Muris, 2002). Two earlier brain imaging studies are noteworthy in this context. In a PET study by Wik, Fredrikson, and Fischer (1996), animal phobics anticipated the appearance of spiders and snakes in neutral film clips (i.e., displayed an encounter expectancy bias), whereas the non-fearful controls did not. Simultaneously, the phobics displayed reduced primary visual cortex activity compared with the non-fearful controls, which was interpreted as a "neurophysiological correlate of avoidant anticipatory coping" (p. 267). Yet, it remains unclear whether reduced visual cortex activity really is a specific correlate of phobogenic expectancies or of other components of the fear response (e.g., subjective feeling state) because expectancies and brain activity were not directly related in this study. Similarly, the anticipation of the presentation of spiders in another study (Straube, Mentzel, \& Miltner, 2007) did not allow for taking these different components apart.

In the present study, therefore, we put subjective expectancies and neural activations into direct relation and asked whether the animal phobics' overestimation (i.e., irrationality) of the likelihood of encountering spiders relates to (a) overactivation of the fear circuit (with the amygdala as a key structure), (b) reduced self-regulation capacities, and/or (c) reduced visual attention. Spider phobics and control participants viewed pictures of spiders, snakes, and birds, and were simultaneously given background information suggesting a low, medium, or high likelihood of encounters with these animals. The participants were subsequently asked to provide an 
estimate regarding their own risk to encounter the animals in this context. Both fMRI and behavioral measures were acquired during the task in order to determine the neural processes (i.e., emotional brain systems) underlying the encounter expectancy bias (Aue \& Hoeppli, 2012) in spider phobia. ${ }^{2}$

Specifically, we investigated whether exaggerated encounter expectancies for spiders in spider phobics can be related to distinctive neural responding (i.e., neural responding that is absent for encounter expectancies regarding snakes and birds in spider phobics and for encounter expectancies regarding all animal categories in the control group). From the existing literature on threat perception, high encounter estimates for spiders in spider phobia might be predicted to be associated with high activity of the fear circuit (e.g., the amygdala). However, it is also conceivable that cognitive or emotion regulation deficits are at the origin of exaggerated expectancies in spider phobia. In the latter case, we would expect, among other effects, distinctive alterations in the recruitment of prefrontal areas (e.g., Carlsson et al., 2004; Hermann et al., 2009). Finally, we tested whether, as suggested by work conducted by Wik and collaborators (1996), abnormal visual cortex activity would be a specific correlate of the encounter expectancy bias in spider phobia.

\section{Method \\ Participants}

In total, 36 individuals (all female, 18 spider phobics), aged between 19 and 44 years $(M=25.8, S D=5.79)$ took part in the study. One participant in the control group was excluded from all analyses because she reported to have misunderstood the task instructions. For further characteristics of the two groups of participants, refer to Aue and Hoeppli (2012).

\section{Stimuli}

Stimuli consisted of 30 pictures displaying spiders and 30 pictures displaying snakes, all taken from a recently created picture base (Dan-Glauser \& Scherer, 2011). Spiders and snakes covered virtually the whole picture and were located on trees, in front of trees, or on the grass. Spider and snake pictures were matched for valence, $t(58)=0.08, n s(M s=3.1$ and $3.1 ; S D s=0.94$ and 0.95 , respectively; scale range: 1 [very unpleasant] to 9 [very pleasant]) and for arousal ratings, $t(58)=0.03, n s(M s=6.1$ and $6.1 ; S D s=0.88$ and 0.75 , respectively; scale range: 1 [not arousing at all] to 9 [very arousing]), as assessed in an earlier study (Dan-Glauser \& Scherer, 2011). Thirty additional pictures displaying birds were collected from the Internet.

\footnotetext{
${ }^{2}$ We also acquired peripheral nervous system activity. Effects unrelated to expectancies are presented elsewhere (Aue, Hoeppli, \& Piguet, 2012).
}

\section{Procedure}

Upon participants' arrival at the laboratory, written informed consent was obtained in accordance with the Helsinki Declaration of Human Rights (World Medical Association, 1999) and regulations approved by the local ethics committee. Participants imagined they were visiting different locations in a forest, with the possibility of encountering different animals (spiders, snakes, or birds) at these locations. On each trial, background information was provided in the form of the frequency at which two forest officials had encountered one of these animals at the depicted location. Participants then had to form and provide their own encounter expectancy, followed by an intensity rating for the fear they would experience in case of such an encounter. The detailed trial sequence is displayed in Fig. 1 (see also Aue \& Hoeppli, 2012). Responses were given by pressing two buttons on an MRIcompatible button box $(\mathrm{HH}-1 \times 4-\mathrm{CR}$, Current Designs Inc., USA), which moved a slider in steps of $6.25 \%$ across a horizontal scale on the screen (17-point scale; starting point in each trial: $50 \% ; 0 \%$ [no risk of encounter/no fear at all]; $100 \%$ [absolute certainty of encounter/maximal fear]). Visual stimuli were presented on a back projection screen inside the MRI scanner bore with an LCD projector (CP-SX1350, Hitachi, Japan).

\section{fMRI data acquisition and image processing}

Transverse $T_{2}{ }^{*}$-weighted echo-planar images (EPIs) were acquired with a 3T scanner (Trio TIM, Siemens, Germany) using the product 12 channel head coil. Participants wore earplugs to attenuate scanner noise; head movement was restricted by a vacuum pillow.

Structural images were acquired with a $T_{1}$-weighted $3 \mathrm{D}$ sequence (MPRAGE, TR/TI/TE = 1900/900/2.27 ms, flip angle $=9$ degrees, PAT factor $=2$, voxel dimensions: $1 \mathrm{~mm}$ isotropic, $256 \times 256 \times 192$ voxels). Functional images were acquired with a susceptibility-weighted EPI sequence $(\mathrm{TR} / \mathrm{TE}=2000 / 30 \mathrm{~ms}$, flip angle $=80$ degrees, PAT factor $=2,64 \times 64$ pixel, $3.2 \times 3.2$ $\mathrm{mm}, 36$ slices, $3.2 \mathrm{~mm}$ slice thickness, 20\% slice gap). An automatic shimming procedure was performed to minimize inhomogeneities of the static magnetic field. At the beginning of each session, image acquisition started after the recording of three dummy volumes to avoid $T_{1}$ saturation effects.

MRI data were pre-processed and analyzed using SPM8 (Wellcome Dept. of Imaging Neuroscience, London, UK; http://www.fil.ion.ucl.ac.uk/spm). Functional images were spatially realigned to the first volume by rigid body transformation, corrected for time differences in slice acquisition by using the middle slice in time as reference, spatially normalized to the standard Montreal Neurological Institute (MNI) EPI template to permit group analysis, resampled to an isotropic voxel size of $3 \mathrm{~mm}$, and spatially 


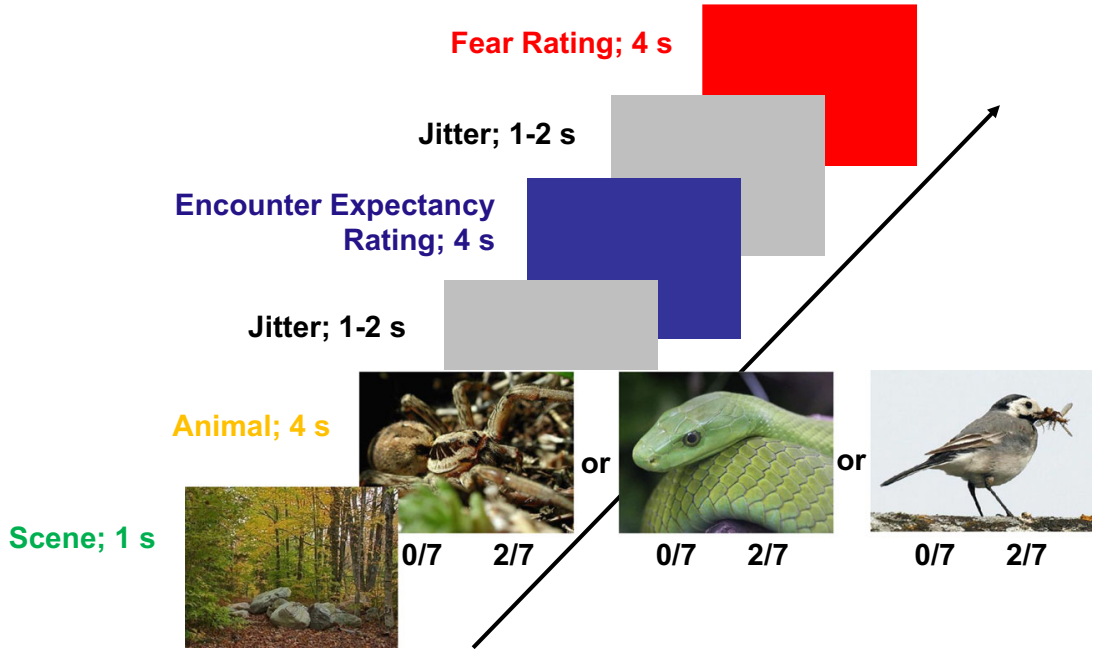

Fig. 1 Trial sequence. In each trial, participants saw a picture of a forest location ( $1 \mathrm{~s}$ ), followed by a picture of an animal (spider, snake, or bird; 4 s). When they saw the animal, participants were simultaneously given background information about the number of times two forest officials had encountered this animal out of the total number of times they had visited the same forest location (e.g., 0/7 and 2/7; displayed below the animal pictures). The objective probabilities (= average of the two likelihoods given as background information) were equal for all three animals.

smoothed with an isotropic 8-mm full-width at half-maximum (FWHM) Gaussian kernel (Friston et al., 1995).

\section{fMRI analysis}

We performed standard analyses using the general linear model (GLM) as implemented in SPM8, where event-related signal changes were modeled separately for each participant. For each block of the experimental task, we specified a linear model that included 10 event types, corresponding to each picture onset and subsequent rating periods (see Fig. 2). The length of the first four events was set to 0 (scene and animal pictures) because the perceptual processes that are supposed to happen during these events have a clear onset. The length of the six events belonging to rating periods, in contrast, was set to $4 \mathrm{~s}$,
The participants' task, then, was to estimate the likelihood that they would encounter the same animal if they were at the same location in the forest by using a 17-point scale (ranging from $0 \%$ [no risk of encounter at all] to $100 \%$ [absolute certainty of encounter]). They were then asked to provide an estimate of the fear they would experience in this scenario on a 17point scale (scale ranging from $0 \%$ [no fear at all] to $100 \%$ [extreme, paralyzing fear])

because the processes expected to happen in these periods do not have obvious onsets (when exactly do participants come to a conclusive decision that they express in their ratings?).

For the encounter expectancy rating events, we added three parametric modulators (one for each animal) that described the modulation of the hemodynamic response in the expectancy rating phase by the subjective expectancy of encountering the animal (encounter expectancy estimate, as indicated in the behavioral response). In the parametric design in SPM, different levels of the expectancy ratings are represented numerically within one extra column (within each participant). Thus, in the expectancy rating phase, each participant had two predictors for each animal condition (see Fig. 2): an overall effect for animal and, in addition, the parametric modulator that described the modulation of the magnitude of the hemodynamic
Fig. 2 Model specification in SPM. Numbers in parentheses refer to the number of events modeled per time point/interval. + 3 PM refers to the number of parametric modulators added to the model. See fMRI data analysis for details

\section{Scene (1) \\ Animal (3)}

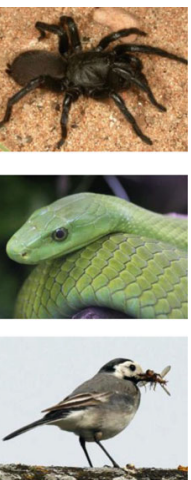

Encounter Expectancy

Rating (3 + 3 PM)
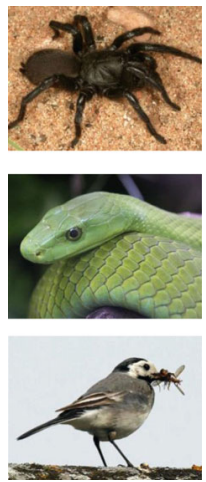

Modulation by Encounter Expectancy Rating (Behavior)
Fear Rating (3)

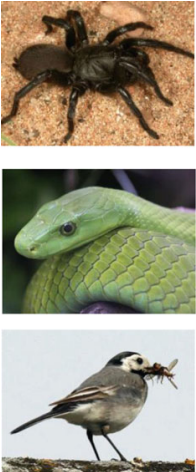


response within each animal category by the expectancy rating score as exhibited in the participant's behavioral data. (We also analyzed parametric modulations for the animal picture presentation phase to test whether neural activity during picture viewing was able to predict phobia-specific variations in encounter expectancies. However, because we did not find any significant effect in these analyses, they are not described here.)

A constant covariate representing the session-specific mean over scans, as well as six motion correction parameters extracted from the realignment procedure, were also added. The model included a high-pass filter of $128 \mathrm{~s}$ to remove lowfrequency drift of the scanner and first-order auto-regressive corrections for auto-correlation between scans. Effects at each brain voxel were estimated by using a least squares algorithm.

Data were analyzed in two steps. First, we investigated neural responses upon initial viewing of the animals (animal picture onset) in order to situate our own findings with respect to the existing literature (Step 1; data quality check). Second, and most important, we investigated our major research question, namely, how neural responses relate to irrational subjective expectancies of encounters with spiders in spider phobics (Step 2). Data modeling and analyses related to these two steps were conducted as follows.

Step 1 Neural responses upon initial viewing of the animals (data quality check).

To allow the comparison of our own findings with earlier studies, we performed a whole-brain analysis in which we contrasted responses to spiders versus snakes during the presentation of the pictures (animal presentation phase) in phobics versus controls. By doing this, we identified particularities of spider phobics' initial perceptual responses toward spiders (relative to fear-relevant but non-phobogenic animals): These responses should neither be seen for snakes in this group, nor should they appear in controls. In order to avoid alpha inflation and to save space (for statistically determined critical cluster sizes, see Step 2), we only report significant clusters containing at least 30 contiguous voxels at a $p<.001$ (uncorrected). All parametric maps were rendered on sections of the average $T_{1}$-weighted template brain of all participants.

\section{Step 2 Neural responses related to phobia-specific expectancies.}

\section{Modulation of neural activity during expectancy rating phase as a function of subjective likelihood of encounter}

Whole-brain analysis With Step 2, we investigated our major research question, namely, whether irrational expectancies regarding spider encounters in spider phobics are characterized by specific neural signatures. As outlined in the introduction, spider phobics have been found to exhibit distinctive subjective expectancies concerning the likelihood of encountering spiders (Aue \& Hoeppli, 2012; de Jong \& Muris, 2002). Consequently, we predicted the existence of brain areas whose activity displays selective modulation by subjective encounter expectancies for spiders in spider phobics; these modulations should not be a characteristic of "normal" variations in expectancies (e.g., encounter expectancies for snakes in spider phobics or encounter expectancies for spiders in controls).

To investigate the neural correlates of subjective expectancies in spider phobia, therefore, we compared the parametric modulations of brain activity during the expectancy rating phase as a function of encounter likelihood reported (behavior rating) for spiders versus snakes $\left(\bmod _{\mathrm{SP}}-\bmod _{\mathrm{SN}}\right)$ in each phobic and control participant. Subsequently, we calculated a second-level $t$ test to identify areas displaying group differences regarding this parametric modulation contrast performed within each participant.

In order to test whether activity in the identified clusters displays phobia-specific expectancy modulations (rather than stemming from particularities in phobia-unspecific modulations, e.g., concerning the control groups' responses to snakes), we extracted the contrast estimates regarding the parametric modulations for spiders, snakes, and birds for each participant (averaged across all voxels constituting a cluster) and subjected them to an analysis of variance (ANOVA) with the factors Animal (spider, snake, bird) and Group (phobic, control). The existence of phobia-specific modulations was demonstrated by significant Group $\times$ Animal interactions, followed by post hoc Tukey tests demonstrating that it was really the spider phobics' modulator for spiders that made up the effect (i.e., differed from the other parametric modulators).

In order to avoid alpha inflation, we only report significant clusters containing at least 19 contiguous voxels at $p<.005$. This critical cluster size was calculated by a Monte Carlo simulation with 10,000 iterations, assuming some interdependence between voxels (8-mm FWHM), resulting in a corrected whole-brain $p$ value of .05 . The application of such comparably liberal alpha thresholds at the voxel level and the whole-brain level seems to be appropriate in the investigation of the subtle affective/cognitive processes with imprecise temporal dynamics that we study here (e.g., in order to avoid massive beta error inflation; see Lieberman \& Cunningham, 2009, for a detailed description on how to balance Type I and Type II errors in cognitive neuroscience). Notably, these alpha levels were actually applied to a double statistical test (i.e., contrast on a previously determined linear parametric relationship). All parametric maps were rendered on sections of the average $T_{1}$-weighted template brain of all participants.

Region of interest (ROI) analysis The whole-brain approach for the expectancy-related parametric modulations was complemented by an ROI analysis for left and right amygdala. 
We determined these ROIs according to the automated anatomical labeling approach of activations (Tzourio-Mazoyer et al., 2002) by using the MarsBaR ROI toolbox for SPM (Brett, Anton, Valabregue, \& Poline, 2002). ROIs were applied as masks to the model specified for the whole-brain analysis. Contrast estimates for these ROIs were then extracted and subjected to an ANOVA with the factors Animal (spider, snake, bird) and Group (phobic, control).

\section{Link between degree of phobia-specific modulation of brain activity by subjective expectancy level and magnitude of encounter expectancy bias displayed}

Whereas the aforementioned analyses allowed the identification of phobia-specific expectancy modulations in brain activity, they did not yield any conclusion as to whether these modulations were directly implicated in the degree of irrationality (i.e., overestimation) displayed. This is because high expectancies of encounters were usually associated with background information suggesting a high likelihood of an encounter. Therefore, the first analysis did not allow us to conclude whether the presented results were related to bias (i.e., irrationality) or simply to high fear.

We hypothesized that the selective modulation of brain activity by encounter expectancies for phobogenic material was directly related to the degree of expectancy bias displayed (i.e., the magnitude of a phobic participant's irrationality or overestimation of encounter expectancies for spiders with respect to the objective background information given) in at least some of the areas identified earlier. To test this hypothesis, we adopted a between-participants approach and correlated phobia-specific expectancy-related modulations in the areas identified in the whole-brain analysis (i.e., contrast estimates for the parametric modulations) with the magnitude of individual expectancy bias (i.e., the degree of overestimation of encounter likelihood for spiders revealed in the behavior) within each group of participants. ${ }^{3}$

Specifically, for each individual, the mean encounter expectancy rating for snakes was subtracted from the mean encounter expectancy rating for spiders (indexing the specific

\footnotetext{
${ }^{3}$ At first glance, one might consider using the deviation of subjective expectancies from the objective risk information as a parametric modulator of the brain response in the GLM model - similar to the procedure described previously for the absolute subjective expectancies. However, an important observation we made was that deviations in encounter expectancies for spiders in phobics were not independent from the objective risk of encountering them (see Aue \& Hoeppli, 2012, for details). Specifically, our participants particularly overestimated the risk of encountering spiders when the given objective likelihood was low. Consequently, a within-participant parametric approach for deviations in expectancies might confound effects of the overall expectancy level versus the relative expectancy deviation because high deviations would always (or most of the time) imply low subjective expectancy, and low deviations would imply high expectancy. To rule out the possibility of such confounding effects, we added the between-participants correlation analysis.
}

expectancy bias for spiders). We then calculated betweenparticipants Pearson product-moment correlation coefficients for the association between this behavioral expectancy bias, on the one hand, and the expectancy modulation effects revealed in brain activity (as identified in the whole-brain analysis described earlier), on the other hand, in both spider phobics and controls. Again, responses to snakes were included as a reference category to ensure that observed effects were phobia specific. Identified correlations were tested for significance and additionally transformed into Fisher's $Z$ values and tested for group differences ( $p<.05$, one-tailed).

\section{Results}

Behavioral data

\section{Fear and encounter expectancy ratings}

Figure 3 displays the participants' fear (manipulation check) and encounter expectancy ratings. Spider phobic and control participants both displayed fear in response to evolutionary threat (spiders and snakes) but not to birds. In addition, spider phobics reported significantly greater fear during the spider trials than during snake and bird trials, and also greater fear during the spider trials than did the control group. The 2 (Group) $\times 3$ (Animal) ANOVA conducted on the fear ratings showed a main effect for Group, $F(1,33)=19.59, p<.001$, a main effect for Animal, $F(2,66)=151.17, p<.001$, and a significant interaction of Group and Animal, $F(2,66)=46.41$, $p<.001$.

Moreover, spider phobics were characterized by a significant expectancy bias for encounters with spiders. Despite the same background information being presented (objective probability), they estimated the risk of encountering spiders to be much higher than the risk of encountering snakes and birds. In controls, no such bias was observed. The 2 (Group) $\times$ 3 (Animal) ANOVA conducted on the expectancy ratings showed no main effect for Group, $F(1,33)=0.43, n s$, a main effect for Animal, $F(2,66)=9.78, p<.0005$, and a significant interaction of Group and Animal, $F(2,66)=7.06, p<.005$. More detailed descriptions and discussions of the behavioral data in this study can be found elsewhere (Aue \& Hoeppli, 2012).

\section{MRI data}

Neural responses upon initial viewing of the animals (data quality check)

To compare our own findings with those of earlier studies, we performed a whole-brain analysis in which we contrasted responses to spiders versus snakes during the presentation of the 
Fig. 3 Participants' fear and encounter expectancy ratings. Error bars depict standard errors

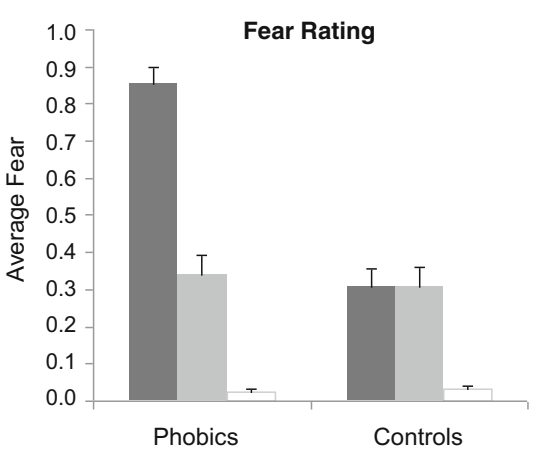

pictures (animal presentation phase) in phobics versus controls. Figure 4 shows the brain regions exhibiting group differences in activity for the contrast "spider versus snake" during the animal picture presentation phase. Separate analyses for phobics and controls revealed that increased activation in response to spiders in these areas was limited to the phobic group; controls exhibited no differential responses to the two animal categories.

Enhanced activity in spider phobics spanned almost the whole brain, including, in particular, the amygdala, visual cortices, thalamus, insula, medial and lateral prefrontal areas, and both dorsal and ventral striatum. The contrast "spider versus bird" produced almost identical results (not described here; see Supplementary Figure 1 for activation clusters). Finally, the contrast "snake versus bird" did not reveal any group differences in the animal presentation phase. Together, these data confirm a selective and robust recruitment of the fear networks in response to spider stimuli in spider phobics, and
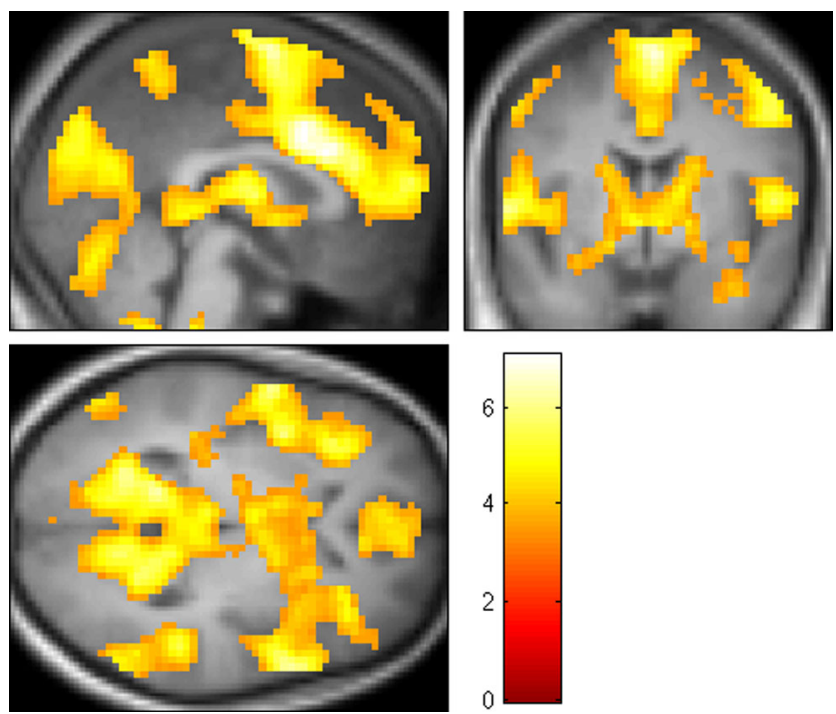

Fig. 4 Areas displaying group differences upon viewing spiders versus snakes (phobics $>$ controls); animal picture presentation phase. $p<.001 ; \mathrm{k}$ $\geq 30$ voxels thus clearly validate the reliability of our paradigm and our population selection.

\section{Neural responses related to phobia-specific expectancies}

Modulation of neural activity during expectancy rating phase as a function of subjective likelihood of encounter

Whole-brain analysis

We predicted the existence of brain areas that display selective modulation by subjective encounter expectancies for spiders in spider phobics, modulations that should not be a characteristic of "normal" variations in expectancies (e.g., encounter expectancies for snakes in spider phobics or encounter expectancies for spiders in controls). The comparison of the parametric expectancy effect between the two fear-relevant categories $\left(\bmod _{\mathrm{SP}} \mathrm{vs} . \bmod _{\mathrm{SN}}\right)$ revealed nine clusters showing differential modulation for spider phobics versus controls (whole-brain analysis; see Fig. 5; Table 1). These were located in the precuneus, IPFC (right: dlPFC; left: both ventromedial prefrontal cortex [vmPFC] and dIPFC), occipital cortex, and premotor cortex (PMC)/supplementary motor area (SMA). Separate analyses for each group revealed that these effects could be fully attributed to the differential response of phobics to spiders versus snakes, whereas there was no difference in controls.

To ensure that these effects were related to particularities in spider phobics' encounter expectancies for spiders (rather than snakes), we also extracted the parameter estimates from these nine clusters obtained with the parametric analysis (corresponding to the slope of the modulatory effect), and then calculated an ANOVA with the factors Animal (spider, snake, bird) and Group (phobic, control) for each region. All analyses revealed a significant interaction of Animal and Group (see Table 2, top). Post hoc Tukey tests demonstrated that the effects indeed mainly reflected a selective modulation by expectancy level for spiders in spider phobics (see Fig. 6 for an exemplary pattern in the precuneus [first cluster specified in Tables 1 and 2]; Supplementary Figure 2 displays modulation 

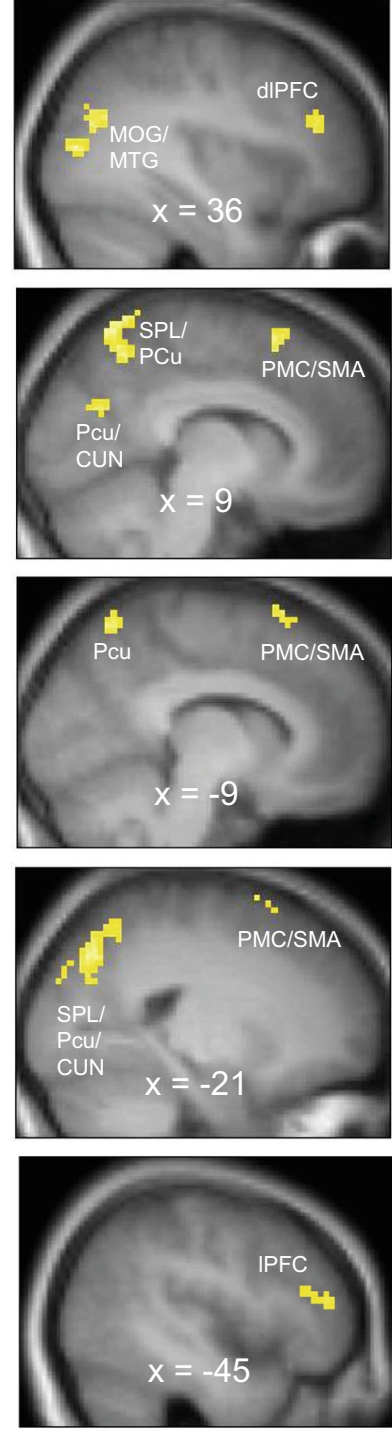

Fig. 5 Modulation of neural activity as a function of subjective likelihood of encounter (contrast $\bmod _{\mathrm{SP}}<\bmod _{\mathrm{SN}}$; phobics $>$ controls); expectancy rating phase. $p<.005 ; \mathrm{k} \geq 19$ voxels. $\mathrm{CUN}=$ cuneus; $\mathrm{d} I \mathrm{PFC}=$ dorsolateral prefrontal cortex; $1 \mathrm{PFC}=$ lateral prefrontal cortex; $\mathrm{MOG}=$ middle occipital gyrus; $\mathrm{MTG}=$ middle temporal gyrus; $\mathrm{PCu}=$ precuneus; $\mathrm{PMC}=$ premotor cortex; SMA $=$ supplementary motor area; $\mathrm{SPL}=$ superior parietal lobule

patterns for all clusters obtained). Only in the left IPFC and the left PMC/SMA did expectancy effects arise because of particular modulations for spiders and for snakes in spider phobics. Again, there was no effect in controls.

Remarkably, across all clusters, the effects observed in phobics involved a negative relationship between neural activity during expectancy rating and actual expectancy level - a relationship that was unique for spider trials (i.e., no such effect was seen for snakes or birds in this group). In other words, the higher the phobics estimated the risk to encounter a spider, the greater the reduction (or the smaller the increase) in activity they displayed in the precuneus,
Table 1 Areas displaying modulation of activity by subjective likelihood of encounter (contrast $\bmod _{\mathrm{SP}}<\bmod _{\mathrm{SN}}$; phobics $>$ controls); expectancy rating phase

\begin{tabular}{|c|c|c|c|c|c|c|}
\hline \multirow[t]{2}{*}{ Region } & \multirow[t]{2}{*}{$\mathrm{k}$} & \multirow[t]{2}{*}{$t$} & \multirow[t]{2}{*}{$p_{\text {uncorr }}$} & \multicolumn{3}{|c|}{ MNI Coordinates } \\
\hline & & & & $x$ & $y$ & $z$ \\
\hline \multirow[t]{3}{*}{ SPL, PCu } & \multirow[t]{3}{*}{108} & 3.96 & $<.001$ & 6 & -61 & 55 \\
\hline & & 3.77 & $<.001$ & 12 & -61 & 67 \\
\hline & & 3.24 & $<.005$ & 21 & -70 & 55 \\
\hline \multirow[t]{3}{*}{$\mathrm{SPL}, \mathrm{PCu}, \mathrm{CUN}$} & \multirow[t]{3}{*}{136} & 3.58 & $<.001$ & -21 & -76 & 40 \\
\hline & & 3.45 & $<.001$ & -24 & -61 & 55 \\
\hline & & 3.26 & $<.005$ & -24 & -76 & 31 \\
\hline \multirow[t]{2}{*}{$\mathrm{PCu}, \mathrm{CUN}$} & \multirow[t]{2}{*}{27} & 3.50 & $<.001$ & 9 & -70 & 25 \\
\hline & & 2.94 & $<.005$ & 0 & -82 & 25 \\
\hline \multirow[t]{2}{*}{$\mathrm{PCu}$} & \multirow[t]{2}{*}{23} & 3.42 & $<.001$ & -9 & -64 & 61 \\
\hline & & 2.83 & $<.005$ & -3 & -55 & 61 \\
\hline dlPFC & 64 & 3.59 & $<.001$ & 33 & 41 & 22 \\
\hline \multirow[t]{2}{*}{ 1PFC } & \multirow[t]{2}{*}{56} & 3.76 & $<.001$ & -48 & 35 & 16 \\
\hline & & 3.25 & $<.005$ & -39 & 23 & 19 \\
\hline \multirow[t]{3}{*}{ MOG, MTG } & \multirow[t]{3}{*}{70} & 4.21 & $<.0001$ & 42 & -79 & 10 \\
\hline & & 3.55 & $<.001$ & 33 & -67 & 25 \\
\hline & & 3.25 & $<.005$ & 30 & -79 & 10 \\
\hline \multirow[t]{3}{*}{ PMC, SMA } & \multirow[t]{3}{*}{92} & 3.67 & $<.001$ & 18 & 26 & 37 \\
\hline & & 3.64 & $<.001$ & 18 & 17 & 64 \\
\hline & & 3.38 & $<.001$ & 9 & 17 & 58 \\
\hline \multirow[t]{2}{*}{ PMC, SMA } & \multirow[t]{2}{*}{26} & 3.39 & $<.001$ & -15 & 17 & 67 \\
\hline & & 3.06 & $<.005$ & -21 & 8 & 70 \\
\hline
\end{tabular}

Note. $n=18$ in each group. $\mathrm{k}=$ cluster size in number of voxels; $M N I$ Coordinates Montreal Neurological Institute coordinates referring to the maximally activated voxel(s) within each cluster (up to 3 per cluster). $C U N$ cuneus, $d l P F C$ dorsolateral prefrontal cortex, $l P F C$ lateral prefrontal cortex, $M O G$ middle occipital gyrus, $M T G$ middle temporal gyrus, $P C u$ precuneus, $P M C$ premotor cortex, SMA supplementary motor area, $S P L$ superior parietal lobule.

IPFC, occipital cortex, and PMC/SMA. ${ }^{4}$ Activity in the left (but not in the right) IPFC and in the left (but not in the right) PMC/SMA showed an additional positive relationship with expectancy level for snakes in spider phobics.

ROI analysis

Modulation parameters were also extracted from the left and right amygdala and subjected to an ANOVA with the factors Animal (spider, snake, bird) and Group (phobic, control). The critical interactions Animal $\times$ Group failed to reach significance (see Table 2, bottom), indicating that

\footnotetext{
${ }^{4}$ Importantly, we also checked for the mean spider activities during the expectancy phase (unaffected by the expectancy modulations). Only the $\mathrm{PCu}, \mathrm{CUN}$, and dlPFC clusters were characterized by an overall decrease in activity for spiders in spider phobics. The other clusters did not show any change in activity (SPL, PCu, CUN; $1 \mathrm{PFC}$; left and right PMC, $\mathrm{SMA}$ ), or even showed an overall increase (PCu; SPL, PCu; MOG, MTG).
} 
Table 2 ANOVA results for the modulation of activity as a function of subjective likelihood of encounter (for areas displayed in Table 1 [whole-brain analysis], top, and for amygdala [region of interest] bottom); expectancy rating phase

\begin{tabular}{|c|c|c|c|c|c|c|c|c|c|}
\hline \multirow[t]{2}{*}{ Region } & \multicolumn{2}{|l|}{ Group } & \multicolumn{2}{|l|}{ Animal } & \multicolumn{2}{|c|}{ Group $\times$ Animal } & \multicolumn{3}{|c|}{ MNI Coordinates } \\
\hline & $F(1,33)$ & $p$ & $F(2,66)$ & $p$ & $F(2,66)$ & $p$ & $x_{\max }$ & $y_{\max }$ & $z_{\max }$ \\
\hline \multicolumn{10}{|c|}{ Areas identified by contrast $\bmod _{\mathrm{SP}}<\bmod _{\mathrm{SN}}$; phobics $>$ controls; whole-brain analysis } \\
\hline $\mathrm{SPL}, \mathrm{PCu}$ & 1.14 & ns & 3.07 & .05 & 8.15 & $<.0011^{\mathbf{A}, \mathbf{B}, \mathbf{C}, D}$ & 6 & -61 & 55 \\
\hline SPL, PCu, CUN & 0.72 & ns & 1.39 & ns & 6.89 & $<.005^{\mathrm{A}, \mathrm{C}}$ & -21 & -76 & 40 \\
\hline $\mathrm{PCu}, \mathrm{CUN}$ & 1.70 & ns & 1.95 & ns & 5.53 & $<.01{ }^{\mathbf{A}, \mathbf{C}, D}$ & 9 & -70 & 25 \\
\hline $\mathrm{PCu}$ & 1.76 & ns & 1.06 & ns & 5.85 & $<.005^{\mathrm{A}, \mathrm{C}}$ & -9 & -64 & 61 \\
\hline dlPFC & 0.89 & ns & 4.34 & $<.05$ & 6.24 & $<.005^{\mathrm{A}, C, \mathrm{D}}$ & 33 & 41 & 22 \\
\hline 1PFC & 0.27 & ns & 3.10 & .05 & 6.76 & $<.005^{\mathrm{A}, \mathrm{E}}$ & -48 & 35 & 16 \\
\hline MOG, MTG & 1.82 & ns & 1.94 & ns & 6.21 & $<.005^{\mathbf{A}, \mathbf{B}, \mathbf{C}, D}$ & 42 & -79 & 10 \\
\hline PMC, SMA & 1.58 & ns & 4.90 & $<.05$ & 8.05 & $<.001$ A,C,D & 18 & 26 & 37 \\
\hline PMC, SMA & 0.82 & ns & 3.28 & $<.05$ & 5.48 & $<.01 \mathrm{~A}, D, F$ & -15 & 17 & 67 \\
\hline \multicolumn{10}{|c|}{ Region of interest analysis } \\
\hline Left amygdala & 7.15 & $<.05$ & 1.52 & ns & 0.15 & ns & & & \\
\hline Right amygdala & 6.33 & $<.05$ & 0.98 & ns & 0.57 & ns & & & \\
\hline
\end{tabular}

Note. $n=18$ in each group. Bold: $p<.05$ (two-tailed); italics: $p<.10$ (two-tailed). MNI Coordinates Montreal Neurological Institute coordinates referring to the maximally activated voxel in the cluster (remember, however, that extracted parameter estimates for parametric modulations in each cluster refer to the average effect observed in all of its voxels). ANOVA analysis of variance, $C U N$ cuneus, $d I P F C$ dorsolateral prefrontal cortex, $I P F C$ lateral prefrontal cortex, $M O G$ middle occipital gyrus, $M T G$ middle temporal gyrus, $P C u$ precuneus, $P M C$ premotor cortex, $S M A$ supplementary motor area, $S P L$ superior parietal lobule. Letters A to F refer to significance levels in post hoc Tukey tests: A = phobic spider vs. phobic snake; $\mathrm{B}=$ phobic spider vs. phobic bird; $\mathrm{C}=$ phobic spider vs. control spider; $\mathrm{D}=$ phobic spider vs. control bird; $\mathrm{E}=$ phobic snake vs. control snake; $\mathrm{F}=$ phobic snake vs. phobic bird.

differences in encounter expectancy levels were not predicted by the magnitude of amygdala activation.

Link between degree of phobia-specific modulation of brain activity by subjective expectancy level and magnitude of encounter expectancy bias displayed We further predicted the selective modulation of brain activity by encounter expectancies for phobogenic material to be directly related to the degree of expectancy bias (i.e., irrationality) displayed in at least

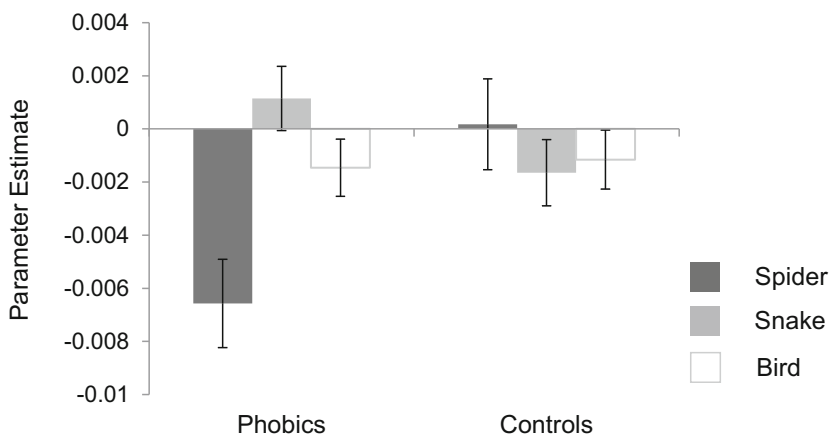

Fig. 6 Modulation of precuneus activity as a function of subjective likelihood of encounter (contrast $\bmod _{\mathrm{SP}}<\bmod _{\mathrm{SN}}$; phobics $>$ controls); expectancy rating phase. A similar activation pattern was observed in the lateral prefrontal cortex, occipital cortex, and right premotor cortex/ supplementary motor area (see Supplementary Figure 2 for details) some of the areas identified earlier. Table 3 shows the between-participants Pearson product-moment correlation coefficients between the degree of parametric modulation of brain activity by expectancy ratings, on the one hand, and the magnitude of the expectancy bias for spiders relative to snakes, on the other hand. Specifically, these correlations were calculated between the parametric estimates (i.e., slope) extracted from each of the nine clusters that were modulated by expectancy ratings, on the one hand, and the overall encounter expectancy rating difference (spider vs. snake), on the other hand. All clusters located in the precuneus exhibited a strong relationship with the individual expectancy bias, suggesting a key implication of this structure in phobia-specific deviations of subjective expectancies. In addition, both the right dlPFC and the occipital cortex revealed the same pattern as the precuneus clusters.

\section{Discussion}

Neural responses upon initial viewing of the animals (data quality check)

To situate our own findings with respect to those of earlier studies, we performed a whole-brain analysis in which we contrasted responses to spiders versus snakes during 
Table 3 Correlations between modulation of brain activity by expectancy rating (spider vs. snake; areas corresponding to Table 1) and magnitude of expectancy bias for spiders over snakes

\begin{tabular}{lllllll}
\hline Region & $r_{\text {Phobics }}$ & $r_{\text {Controls }}$ & $Z$ & \multicolumn{3}{c}{ MNI Coordinates } \\
\cline { 5 - 7 } & & & & $x_{\max }$ & $y_{\max }$ & $z_{\max }$ \\
\hline SPL, PCu & $\mathbf{- . 7 2}$ & -.20 & $\mathbf{- 1 . 9 0}$ & 6 & -61 & 55 \\
SPL, PCu, CUN & $\mathbf{- . 5 1}$ & .01 & -1.54 & -21 & -76 & 40 \\
PCu, CUN & $\mathbf{- . 5 9}$ & .08 & $\mathbf{- 2 . 0 4}$ & 9 & -70 & 25 \\
PCu & $\mathbf{- . 5 9}$ & .00 & $\mathbf{- 1 . 8 2}$ & -9 & -64 & 61 \\
dlPFC & $\mathbf{- . 6 5}$ & -.09 & $\mathbf{- 1 . 8 4}$ & 33 & 41 & 22 \\
lPFC & -.35 & -.24 & -0.32 & -48 & 35 & 16 \\
MOG, MTG & $\mathbf{- . 5 9}$ & -.25 & -1.14 & 42 & -79 & 10 \\
PMC, SMA & -.43 & .17 & $\mathbf{- 1 . 7 0}$ & 18 & 26 & 37 \\
PMC, SMA & -.26 & -.02 & -0.66 & -15 & 17 & 67 \\
\hline
\end{tabular}

Note. $n=18$ in each group. Bold: $p<.05$ (two-tailed for $r$; one-tailed for $Z$ ); italics: $p<.10$ (two-tailed for $r$, one-tailed for $Z$ ); gray: $p \geq .10$ (twotailed for $r$; one-tailed for $Z$ ). $Z=$ Fisher's $Z$ transformation, testing the difference between the two group correlations (note that this $Z$ transformation is depicted for the sake of completeness; because the control group did not display any bias, stronger weight was given to the significance of correlations within the phobic group rather than to the contrast of the phobic group's correlations with those of the control group); MNI Coordinates Montreal Neurological Institute coordinates referring to the maximally activated voxel in the cluster (remember, however, that extracted parameter estimates for parametric modulations in each cluster refer to the average effect observed in all of its voxels). CUN cuneus, $d l P F C$ dorsolateral prefrontal cortex, $l P F C$ lateral prefrontal cortex, $M O G$ middle occipital gyrus, $M T G$ middle temporal gyrus, $P C u$ precuneus; $P M C$ premotor cortex, $S M A$ supplementary motor area, $S P L$ superior parietal lobule.

the presentation of the pictures (animal presentation phase) in phobics versus controls. Phobics displayed massively increased activity in many brain areas, including the classic fear circuit (e.g., Davis \& Lang, 2003), emotion regulation systems (e.g., Carlsson et al., 2004; Hermann et al., 2009), and attentional networks (e.g., Pourtois, Schwartz, Seghier, Lazeyras, \& Vuilleumier, 2006), when they were shown pictures of spiders rather than snakes or birds. This pattern constitutes a substantial arousal response and reflects the significant emotional impact of spider stimuli on these participants. Consistent with earlier research (Carlsson et al., 2004; Paquette et al., 2003; Rauch et al., 1995; Schienle et al., 2007; Straube, Mentzel, \& Miltner, 2006, 2007), we found phobic responses to be characterized by increased activity in the amygdala, insula, mOFC, ACC, and midbrain. However, contrary to some earlier findings (e.g., Carlsson et al., 2004; Fredrikson, Wik, Annas, Ericson, \& Stoneelander, 1995; Fredrikson et al., 1993), we did not observe decreases in the lateral OFC, dlPFC, posterior cingulate cortex, and hippocampus.

These findings of increased rather than decreased activity in the regulation network comprising the lateral $\mathrm{OFC}$ and
dlPFC are consistent with Paquette et al.'s (2003) observations and with their proposal that phobics attempt to regulate their exaggerated responses to spiders but apply ineffective strategies. Alternatively, the divergent effects reported for the IPFC areas between studies might be a result of experimental manipulation differences. For example, Carlsson et al.'s (2004) participants only looked at animal pictures, whereas our participants simultaneously received background information about the general risk of encountering the animal presented and had the task to subsequently indicate their personal encounter expectancy estimation. Generally, our participants mentioned having tried to take into account the background information presented; however, when spiders were presented, this may have demanded greater processing resources because of the concomitant influence of other (e.g. fear-related) processes in the phobics.

Somewhat surprisingly, neural activity in the animal picture presentation phase did not affect phobia-specific variations in encounter expectancies as expressed in the subsequent expectancy rating phase (for the specific kind of analysis performed, see fMRI analysis in the Method section). This suggests that our participants' encounter expectancies were shaped independently from their initial response to the pictures displayed. The visual material presented may have prioritized perceptual processing over the processing of expectancies. It is very likely that our participants were able to profoundly process expectancy-related information only when the pictures had finally reduced in size (covering about $1 / 8$ of the screen) and the corresponding rating scale was displayed.

\section{Neural responses related to phobia-specific expectancies}

Our major research question asked whether irrational expectancies regarding spider encounters in spider phobics were characterized by specific neural signatures. In the current study, neural responses during the expectancy rating phase that varied as a function of the imagined expectancies of encountering a phobogenic object (i.e., a spider) included distinctive activity in the precuneus, IPFC, and primary visual cortex. The higher the phobic participants estimated the risk of encountering a spider, the lower the activity in these areas. Importantly, these relationships were not observed in the control group, nor were they seen for snakes or birds in the phobic group. In addition, the negative modulations in the precuneus, right dIPFC, and right primary visual cortex were direct indicators of the magnitude of phobic expectancy bias (i.e., the tendency to overestimate the encounter likelihood for spiders relative to the objective background information). Consequently, differential activation of these brain areas constituted a specific feature of phobic responding.

The regions we found to be related to phobia-specific expectancy modulations in the current study have been associated 
with emotion regulation in previous research (e.g., Cavanna \& Trimble, 2006; see Carlsson et al., 2004; Hermann et al., 2009; Schienle et al., 2007; Wik et al., 1996, for their implications in animal phobia). This suggests that biased expectancies in spider phobia are an important constituent of deficits in emotion regulation processes that are mediated by these areas.

Activity in the dlPFC, for instance, has been linked to topdown control of lower level cognitive processes (e.g., Carlsson et al., 2004; Corbetta \& Shulman, 2002) and proposed to be crucially implicated in the integration of existing knowledge, expectancies, and goals. A core feature of animal phobia is that phobics are aware that their subjective expectancies with respect to the phobic animal are exaggerated and do not correspond to objective risk indicators (as reflected in the Diagnostic and Statistical Manual of Mental Disorders [4th ed., text rev.; American Psychiatric Association, 2000] and the International Classification of Diseases [10th rev.; World Health Organization, 1992] criteria for specific phobia), thus demonstrating discordance between knowledge and expectancies. Higher encounter expectancies for spiders in phobics - implying a frequent experience of threat in the environment - may therefore be the result of a diminished capacity to activate critical neural integration centers.

Consistent with such an interpretation, the precuneus - often in concert with prefrontal cortex activity - has been linked to phenomena such as reflective self-awareness (e.g., Kjaer, Nowak, \& Lou, 2002), adoption of the third-person perspective (e.g., Vogeley et al., 2004), episodic memory retrieval or planning (e.g., Lundstrom, Ingvar, \& Petersson, 2005), or selfregulation (see Cavanna \& Trimble, 2006, for an overview of functions associated with the precuneus). All of these phenomena strongly rely on contextual integration capacities. For this reason, the precuneus constitutes an important convergence zone whose functioning may be impeded in phobogenic situations. Impaired contextual processing of anticipated events and background information due to reduced recruitment of the precuneus may account for unrealistic assessment of threat and encounter likelihood as seen in phobics.

An alternative interpretation of these deviating activities in the dlPFC and precuneus in phobic expectancies consists of pathological memory (e.g., impaired memory for background information displayed; for evidence linking the dlPFC with memory processes, see, for instance, D’Esposito et al., 1995; for evidence linking the precuneus with memory processes, see, for instance, Lundstrom et al., 2005) rather than emotion regulation processes. Given the fact that most research findings on the neural correlates of animal phobia strengthen the idea of reduced regulation capacities (e.g., Carlsson et al., 2004; Hermann et al., 2009), however, our primary interpretation of the data is consistent with the idea of deficiencies in cognitive control and associated integration processes.

The diminished activation in the visual cortex of spiderphobic participants, observed when the imagined risk of encountering spiders was high, might be part of an avoidance response aimed at coping with the situation (Aue, Hoeppli, Piguet, Sterpenich, \& Vuilleumier, 2013; Wik et al., 1996). Because of the inefficiency of coping strategies directed at reducing emotional responses in phobogenic situations, high expectancies of encounters with spiders may only be tolerated by reducing visual attention to the experimental situation. Alternatively, this result may have arisen because higher expectancy ratings implied the direction of visual attention to the right visual hemifield (therefore, reducing activity in the right primary visual cortex).

Impressively, regarding hemispheric lateralization, our effects for encounter expectancy variations were quite symmetric (see Table 1 for details). However, whereas activity in left IPFC was diminished when phobic participants estimated the risk of encountering spiders as high (as in all other clusters displayed), it was not predictive of magnitude of expectancy bias (i.e., the deviations in subjective encounter expectancies from objective background information). Thus, this characteristic reduced activity of high encounter expectancies appeared irrespective of the degree of irrationality in expectancies. A closer look at Table 3 reveals that frontal areas located in the right hemisphere generally exhibited a stronger association with biases in expectancies than did frontal areas located in the left hemisphere. Consequently, in addition to posterior midline structures, right rather than left frontal hemispheric deactivations may be a specific correlate of irrational encounter expectancies in spider phobia.

Notably, we also analyzed parametric modulations for the animal picture presentation phase to test whether neural activity during picture viewing was able to predict phobia-specific variations in encounter expectancies. However, we did not find any expectancy-related activation during that phase. This is possibly because the imagination (and thus the expectations) of an encounter did not happen in, or at least was not restricted to, the animal picture presentation period. Instead, in our task, participants first needed to perceive the animal being presented and to fully process the corresponding background information. They were only then able to integrate this information and subsequently form a personal encounter expectancy. Thus, an expectancy bias should not have arisen instantaneously. It is likely that a complete integration of the information being given happened only in the later expectancy rating phase. This is the moment when participants were prompted to make a conclusive statement about their personal expectation. Therefore, it is not surprising that the behavioral rating (which presents this final outcome expectancy) correlates best with brain activity in the expectancy rating phase.

In addition, phobic individuals often have problems withdrawing attention from phobia-relevant pictures (e.g., Gerdes, Alpers, \& Pauli, 2008), and/or avoiding the phobic triggers being presented (e.g., Rinck \& Becker, 2006). In the current study, there was indeed some indication that our phobic participants withdrew their visual attention from the spider 
pictures approximately $2 \mathrm{~s}$ after stimulus onset (Aue et al., 2013). It is thus possible that they were able to perform the task (i.e., form their personal encounter expectancies) only when the pictures had reduced in size and gave the impression of increasing distance.

The findings in the current study demonstrate some significant differences with respect to earlier studies on spider phobia. Straube et al. (2007) reported spider phobics to display increased activity in the dorsal ACC, insula, thalamus, and bed nucleus of the stria terminalis during the anticipation of the presentation of spider pictures. Yet, none of these areas was associated with the encounter expectancy bias studied here. It is important to note, however, that anticipation in the Straube et al. study was characterized by certainty; whenever the phobics anticipated the apparition of spiders, there was a $100 \%$ likelihood that a spider would subsequently be shown. Therefore, expectancy-related processes may rely on different brain structures in certain versus uncertain situations.

Carlsson et al. (2004) observed reduced activity in the right PFC in spider phobia and suggested that this could reflect the reallocation of processing resources in the service of urgent and immediate response preparation, the latter being mediated by subcortical structures of the fear circuit and orienting processes independent of lateral prefrontal areas. The recruitment of fear systems mediating immediate and direct pursuit of defense in the service of survival might therefore be predicted to inhibit or overrule slower and longer lasting processes that are implicated in the integration of existing knowledge, expectancies, and other goals. Here, however, we found no indication whatsoever that alterations in phobia-related encounter expectancies are associated with increased activity in the amygdala or other structures constituting the fear circuit (Davis \& Lang, 2003). This might be explained by the fact that the amygdala evaluates the general salience of an event (Davis \& Whalen, 2001; Kawashima et al., 1999; Wager, Phan, Liberzon, \& Taylor, 2003), but is not (or is less) dependent on more fine-grained situational factors such as focus of attention (e.g., Straube et al., 2006; Vuilleumier, Armony, Driver, \& Dolan, 2001). In our case, the critical cues signaling general situational salience rather correspond to the presentation of spider images. The viewing of the spider images may be seen as already constituting an encounter with potential threat and may have determined the appraisal of personal relevance. The additional imagination of different probability levels to encounter these animals may have produced modulations in amygdala activity that were too weak to be detectable. Alternative explanations for the absence of expectancyrelated effects in the amygdala may be (a) that amygdala responses are primarily stimulus-driven and typically triggered by sensory cues rather than internal cognitive representations, such as expectancies (see Vuilleumier, Armony, \& Dolan, 2003); (b) that amygdala habituation occurs (e.g., Larson et al., 2006; note, however, that, in our study, amygdala activation during the earlier animal presentation phase did not predict subsequent expectancy ratings either); or (c) that amygdala responding is dampened because of active cognitive processes involved in making expectancy ratings.

Finally, we might be criticized for the rather cognitive nature of our task. For instance, it could be argued that the performance of this type of task relied more strongly on controlled working memory processes than on the automatized processes one usually associates with encounter expectancy bias. Future research should develop innovative new experimental paradigms that make use of more intuitive, and more automatized, mental processes. Existent paradigms of this kind (e.g., de Jong \& Muris, 2002) do not yet allow the determination of the degree of bias or irrationality displayed and also cannot reliably link phobia-specific fine-grained trial-totrial variations in expectancies with abnormal variations in neural activity. One advantage of our task over others in the field is therefore that it enabled the consideration of such finegrained critical variations in expectancies. Moreover, the background information presented allowed the calculation of the objective likelihood of encountering different animals, and a participants' encounter estimate could then be directly related to this objective likelihood, thus determining degree of irrationality. The inclusion of the objective background information further permitted us to keep the mean objective likelihood constant across the different animal categories.

In sum, our data accord with the idea that expectancy biases in spider phobia may reflect deficiencies in cognitive control and contextual integration that are mediated by right frontal and parietal areas. Therapeutic interventions should enable the acquisition of strategies that improve the phobics' capacity to integrate different pieces of information during distressing anticipations of spider confrontations. In the ideal case, spider phobics would then be able to adequately process safety signals, thereby counteracting catastrophic thinking and thus successfully down-regulating their intense fear.

Author Note This research was supported by grant PZ00P1 121590 of the Swiss National Science Foundation to Tatjana Aue.

\section{References}

American Psychiatric Association (2000). Diagnostic and statistical manual of mental disorders (4th ed., text rev.). Washington, DC: Author.

Aue, T., \& Hoeppli, M. E. (2012). Evidence for an encounter expectancy bias in fear of spiders. Cognition and Emotion, 26, 727-736.

Aue, T., Hoeppli, M. E., \& Piguet, C. (2012). The sensitivity of physiological measures to phobic and nonphobic fear intensity. International Journal of Psychophysiology, 26, 154-167.

Aue, T., Lavelle, L. A., \& Cacioppo, J. T. (2009). Great expectations: What can fMRI tell us about psychological phenomena? International Journal of Psychophysiology, 73, 10-16. 
Aue, T., Hoeppli, M. E., Piguet, C., Sterpenich, V., \& Vuilleumier, P. (2013). Visual avoidance in phobia: Particularities in neural activity, autonomic responding, and cognitive risk evaluations. Frontiers in Human Neuroscience, 7, 194.

Bar-Haim, Y., Lamy, D., Pergamin, L., Bakermans-Kranenburg, M. J., \& van IJzendorn, M. H. (2007). Threat-related attentional bias in anxious and nonanxious individuals: A meta-analytic study. Psychological Bulletin, 133, 1-24.

Brett, M., Anton, J. L., Valabregue, R., \& Poline, J. B. (2002, June). Region of interest analysis using an SPM toolbox [Abstract]. Presented at the 8th International Conference on Functional Mapping of the Human Brain, June 2-6, 2002, Sendai, Japan. Available on CD-ROM in NeuroImage 16(2).

Carlsson, K., Petersson, K. M., Lundqvist, D., Karlsson, A., Ingvar, M., \& Öhman, A. (2004). Fear and the amygdala: Manipulation of awareness generates differential cerebral responses to phobic and fear-relevant (but nonfeared) stimuli. Emotion, 4, 340-353.

Cavanna, A. E., \& Trimble, M. R. (2006). The precuneus: A review of its functional anatomy and behavioural correlates. Brain, 129, 564 583.

Corbetta, M., \& Shulman, G. L. (2002). Control of goal-directed and stimulus-driven attention in the brain. Nature Reviews Neuroscience, 3, 201-215.

D’Esposito, M., Detre, J. A., Alsop, D. C., Shin, R. K., Atlas, S., \& Grossman, M. (1995). The neural basis of the central executive system of working memory. Nature, 378, 279-281.

Dan-Glauser, E. S., \& Scherer, K. R. (2011). The Geneva Affective PicturE Database (GAPED): A new 730 picture database focusing on valence and normative significance. Behavior Research Methods, 43, 468-477.

Davey, G. C. L., \& Dixon, A. L. (1996). The expectancy bias model of selective associations: The relationship of judgements of CS dangerousness, CS-UCS similarity and prior fear to a priori and a posteriori covariation assessments. Behaviour Research and Therapy, 34, 235-252.

Davis, M., \& Lang, P. J. (2003). Emotion. In M. Gallagher \& R. J. Nelson (Eds.), Handbook of psychology: Volume 3. Biological psychology (pp. 405-439). New York, NY: Wiley.

Davis, M., \& Whalen, P. J. (2001). The amygdala: Vigilance and emotion. Molecular Psychiatry, 6, 13-34.

de Jong, P. J., \& Muris, P. (2002). Spider phobia: Interaction of disgust and perceived likelihood of involuntary physical contact. Journal of Anxiety Disorders, 16, 51-65.

Dilger, S., Straube, T., Mentzel, H. J., Fitzek, C., Reichenbach, J. R., Hecht, H., \& Miltner, W. H. (2003). Brain activation to phobiarelated pictures in spider phobic humans: An event-related functional magnetic resonance imaging study. Neuroscience Letters, 348, 29-32.

Etkin, A., \& Wager, T. D. (2007). Functional neuroimaging of anxiety: A meta-analysis of emotional processing in PTSD, social anxiety disorder and specific phobia. American Journal of Psychiatry, 164, $1476-1488$

Fredrikson, M., Annas, P., Fischer, H., \& Wik, G. (1996). Gender and age differences in the prevalence of specific fears and phobias. Behaviour Research and Therapy, 34, 33-39.

Fredrikson, M., Wik, G., Annas, P., Ericson, K., \& Stoneelander, S. (1995). Functional neuroanatomy of visually elicited simple phobic fear: Additional data and theoretical analysis. Psychophysiology, 32, 43-48.

Fredrikson, M., Wik, G., Greitz, T., Eriksson, L., Stoneelander, S., Ericson, K., \& Sedvall, G. (1993). Regional cerebral blood-flow during experimental phobic fear. Psychophysiology, 30, 126-130.

Friston, K. J., Holmes, A. P., Worsley, K. J., Poline, J. B., Frith, C. D., \& Frackowiak, R. S. J. (1995). Statistical parametric maps in functional imaging: A general linear approach. Human Brain Mapping, 2, 189-210.
Gerdes, A. B. M., Alpers, G. W., \& Pauli, P. (2008). When spiders appear suddenly: Spider-phobic patients are distracted by task-irrelevant spiders. Behaviour Research and Therapy, 46, 174-187.

Hermann, A., Schäfer, A., Walter, B., Stark, R., Vaitl, D., \& Schienle, A. (2009). Emotion regulation in spider phobia: Role of the medial prefrontal cortex. Social Cognitive and Affective Neuroscience, 4, 257-267.

Kawashima, R., Sugiura, M., Kato, T., Nakamura, A., Hatano, K., Ito, K., \& Nakamura, K. (1999). The human amygdala plays an important role in gaze monitoring: A PET study. Brain, 122, 779-783.

Kjaer, T. W., Nowak, M., \& Lou, H. C. (2002). Reflective self-awareness and conscious states: PET evidence for a common midline parietofrontal core. NeuroImage, 17, 1080-1086.

Larson, C. L., Schaefer, H. S., Siegle, G. J., Jackson, C. A. B., Anderle, M. J., \& Davidson, R. J. (2006). Fear is fast in phobic individuals: Amygdala activation in response to fear-relevant stimuli. Biological Psychiatry, 60, 410-417.

LeDoux, J. E. (Ed.). (1996). The emotional brain. New York, NY: Simon $\&$ Schuster.

Lieberman, M. D., \& Cunningham, W. A. (2009). Type I and Type II error concerns in fMRI research: Re-balancing the scale. Social Cognitive and Affective Neuroscience, 4, 423-428.

Lundstrom, B. N., Ingvar, M., \& Petersson, K. M. (2005). The role of precuneus and left inferior frontal cortex during source memory episodic retrieval. NeuroImage, 27, 824-834.

Mitte, K. (2008). Memory bias for threatening information in anxiety and anxiety disorders: A meta-analytic review. Psychological Bulletin, 134, 886-911.

Mogg, K., \& Bradley, B. P. (2006). Time course of attentional bias for fear-relevant pictures in spider-fearful individuals. Behaviour Research and Therapy, 44, 1241-1250.

Öhman, A., \& Mineka, S. (2001). Fears, phobias, and preparedness: Toward an evolved module of fear and fear learning. Psychological Review, 108, 483-522.

Olatunji, B. O., Sawchuk, C. N., Lee, T., Lohr, J. M., \& Tolin, D. F. (2008). Information processing biases in spider phobia: Application of the Stroop and "White noise" paradigm. Journal of Behavior Therapy and Experimental Psychiatry, 39, 187-200.

Paquette, V., Lévesque, J., Mensour, B., Leroux, J. M., Beaudoin, G., Bourgouin, P., \& Beauregard, M. (2003). 'Change the mind and you change the brain': Effects of cognitive-behavioral therapy on the neural correlates of spider phobia. NeuroImage, 18, 401-409.

Pourtois, G., Schwartz, S., Seghier, M. L., Lazeyras, F., \& Vuilleumier, P. (2006). Neural systems for orienting attention to the location of threat signals: An event-related fMRI study. NeuroImage, 31, 920 933.

Rauch, S. L., Savage, C. R., Alpert, N. M., Miguel, E. C., Baer, L., Breiter, H. C., \& Jenike, M. A. (1995). A positron emission tomographic study of simple phobic symptom provocation. Archives of General Psychiatry, 52, 20-28.

Rinck, M., \& Becker, E. S. (2006). Spider fearful individuals attend to threat, then quickly avoid it: Evidence from eye movements. Journal of Abnormal Psychology, 115, 231-238.

Schienle, A., Schäfer, A., Hermann, A., Rohrmann, S., \& Vaitl, D. (2007). Symptom provocation and reduction in patients suffering from spider phobia. European Archives of Psychiatry and Clinical Neurosciences, 257, 486-493.

Schienle, A., Schäfer, A., Walter, B., Stark, R., \& Vaitl, D. (2005). Brain activation of spider phobics towards disorder-relevant, generally disgust- and fear-inducing pictures. Neuroscience Letters, 388, 1-6.

Straube, T., Mentzel, H. J., \& Miltner, W. H. (2006). Neural mechanisms of automatic and direct processing of phobogenic stimuli in specific phobia. Biological Psychiatry, 59, 162-170.

Straube, T., Mentzel, H. J., \& Miltner, W. H. (2007). Waiting for spiders: Brain activation during anticipatory anxiety in spider phobics. NeuroImage, 37, 1427-1436. 
Tzourio-Mazoyer, N., Landeau, B., Papathanassiou, D., Crivello, F., Etard, O., Delcroix, N., \& Joliot, M. (2002). Automated anatomical labeling of activations in SPM using a macroscopic anatomical parcellation of the MNI MRI single-subject brain. NeuroImage, $15,273-289$.

Vogeley, K., May, M., Ritzl, A., Falkai, P., Zilles, K., \& Fink, G. R. (2004). Neural correlates of first-person perspective as one constituent of human self-consciousness. Journal of Cognitive Neuroscience, 16, 817-827.

Vuilleumier, P., Armony, J. L., Driver, J., \& Dolan, R. J. (2001). Effects of attention and emotion on face processing in the human brain: An event-related fMRI study. Neuron, 30, 829-841.

Vuilleumier, P., Armony, J., \& Dolan, J. (2003). Reciprocal links between emotion and attention. In R. S. J. Frackowiak et al. (Eds.), Human brain function (2nd ed., pp. 419-444). San Diego, CA: Academic Press.

Wager, T., Phan, K. L., Liberzon, I., \& Taylor, S. F. (2003). Valence, gender, and lateralization of functional brain anatomy in emotion:
A meta-analysis of findings from neuroimaging. NeuroImage, 19, 513-531.

Watts, F. N., McKenna, F. P., Sharrock, R., \& Trezise, L. (1986). Colour naming of phobia-related words. British Journal of Psychology, 77, 97-108.

Wik, G., Fredrikson, M., Ericson, K., Eriksson, L., Stoneelander, S., \& Greitz, T. (1993). A functional cerebral response to frightening visual stimulation. Psychiatry Research: Neuroimaging, 50, $15-24$.

Wik, G., Fredrikson, M., \& Fischer, H. (1996). Cerebral correlates of anticipated fear: A PET study of specific phobia. International Journal of Neuroscience, 87, 267-276.

World Health Organization. (1992). International classification of diseases and related health problems (10th rev.). Geneva, Switzerland: Author.

World Medical Association. (1999). Proposed revision of the Declaration of Helsinki. British Medical Journal, 147, 18-22. 